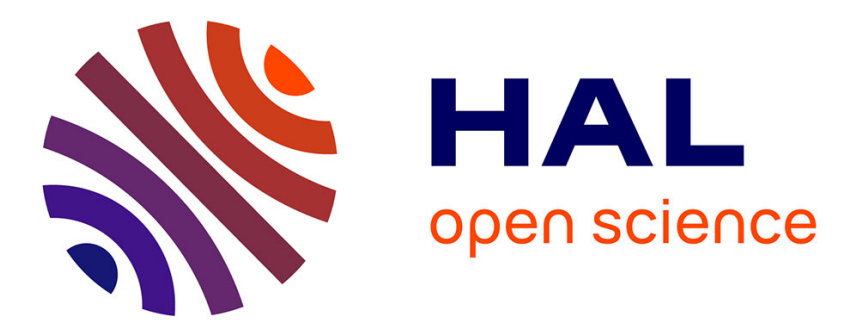

\title{
Réduplication et progression discursive dans la prose beckettienne
}

\author{
Marie-Albane Watine
}

\section{To cite this version:}

Marie-Albane Watine. Réduplication et progression discursive dans la prose beckettienne. Julien Piat, Philippe Wahl. La Prose de Samuel Beckett. Configuration et progression discursives, Presses Universitaires de Lyon, pp.149-160, 2013, 2729708669. hal-01164948

\section{HAL Id: hal-01164948 \\ https://hal.science/hal-01164948}

Submitted on 19 Jun 2015

HAL is a multi-disciplinary open access archive for the deposit and dissemination of scientific research documents, whether they are published or not. The documents may come from teaching and research institutions in France or abroad, or from public or private research centers.
L'archive ouverte pluridisciplinaire HAL, est destinée au dépôt et à la diffusion de documents scientifiques de niveau recherche, publiés ou non, émanant des établissements d'enseignement et de recherche français ou étrangers, des laboratoires publics ou privés.

\section{(ㅇ)(1) $\$$}

Distributed under a Creative Commons Attribution - NonCommercial - NoDerivatives| 4.0 


\section{LA PROSE DE SAMUEL BECKETT CONFIGURATION ET PROGRESSION DISCURSIVES}

Textes réunis et présentés par

Julien Piat \& Philippe Wahl 



\title{
RÉDUPLICATION ET PROGRESSION DISCURSIVE DANS LA PROSE BECKETTIENNE
}

\author{
Marie-Albane Watine
}

«Toute l'œuvre de Beckett sera parcourue de séries exhaustives, c'est-à-dire épuisantes ", écrivait Gilles Deleuze dans sa postface à Quad (Deleuze, 1992, p. 60). En effet, l'un des aspects de cette littérature de l'épuisement ${ }^{1}$ dont Beckett est l'un des meilleurs représentants, c'est la tentative de dire tout le possible par la réalisation de séries combinatoires. D'un point de vue stylistique, cette exhaustion se réalise fréquemment par la répétition avec infime variation dont on trouve tant d'exemples dans la prose de Beckett. Ainsi la célèbre fin de L'Innommable: «je ne sais pas, je ne le saurai jamais, dans le silence on ne sait pas, il faut continuer, je ne peux pas continuer, je vais continuer» $(I, \mathrm{p} .213)$. Ici les tiroirs temporels, les sujets syntaxiques ou les verbes modaux ne cessent de varier autour d'une base lexicale qui, elle, se répète.

Cette répétition avec variation est bien, au niveau microtextuel, une configuration remarquable du style beckettien. Elle assure une forte cohésion discursive en même temps qu'une progression au moins minimale. Mais poussée au terme de sa logique, il arrive que cette écriture de l'infime progression se mue en pur piétinement : stylistiquement, cette absolutisation de la stase se réalise dans la figure de la réduplication, c'est-à-dire de la répétition immédiate et sans variation, comme ici : « on n'en finit pas davantage, je le sais, je le sais. » (M, p. 54) Cette figure, de fréquence remarquable (une occurrence toutes les dix pages pour le corpus que nous étudions), constitue un poste d'observation privilégié puisqu'elle signe par endroits le passage d'une écriture de la progression minimale vers son point ultime, indépassable, qui consiste à redire la même chose exactement sans même se donner la peine de l'infime variation.

Dans cette stase provisoire, il semble que la logorrhée qui impose aux locuteurs beckettiens de continuer de parler ou d'écrire se heurte à l'épuisement de ce qu'il y a à dire, et se résout ponctuellement dans le piétinement. En quelque sorte, la figure semble à la fois dire qu'il faut continuer à dire, mais qu'il n'y a rien de plus à dire. À cet égard, elle comporte une fonction critique touchant aux possibilités du discours et de la littérature.

C'est cette relation entre stase de la progression et fonction critique de la réduplication que nous voulons explorer. Pour aborder ses divers 
avatars et les lignes de son évolution, nous prenons appui sur un corpus large qui va de Premier amour à Compagnie, Mal vu mal dit et Soubresauts, en passant par la trilogie Molloy, Malone meurt, L'Innommable. Après un prologue définitoire, nous distinguerons un premier ensemble de réduplications qui suppose un rapport variable du locuteur à son dire ; un deuxième ensemble de cas inaugure ce qui nous semble une configuration proprement beckettienne, à savoir une progression paradoxalement régressive.

\section{PROLOGUE : APPROCHE DÉFINITOIRE²}

On définira comme réduplication la répétition immédiate et sans terme intercalé d'un quelconque segment textuel. Les niveaux d'analyse concernés vont donc de la simple lexie ("Mais j'étais fatigué, fatigué » $[M$, p. 96]) à l'énoncé complet ("C'est bien, c'est bien. " $[M M$, p. 15]). La réduplication met en série équivalente deux syntagmes qui ont strictement le même rôle fonctionnel ou textuel. Il faut exclure de la réduplication le redoublement infralexématique ${ }^{3}$, qui concerne par exemple les syllabes d'une lexie ; contentons-nous de remarquer que ce piétinement-là semble aussi fasciner Beckett qui en fait grand usage dans les noms propres (Lulu dans Premier amour, Didi et Gogo au théâtre) et $\mathrm{y}$ trouve matière à des calembours scatologiques ${ }^{4}$ : ainsi la " comtesse Caca » de Molloy ( $M$, p. 21), la voix « quaqua " de Comment c'est (CC, p. 167, 175 et 199) ou l'«Acacacacadémie d'Anthropopopométrie » d'En attendant Godot (G, p. 59).

Nous écarterons de notre étude les réduplications qui sont interprétables comme des reformulations. Celles-ci, assez fréquentes dans le corpus beckettien, ajoutent à la suite du segment répété un élément qui le recaractérise, le requantifie ou le redétermine :

(1) sur mon corps, mon corps incapable [...]. (I, p. 23)

(2) Quand ce n'est pas le soir c'est la nuit. Nuit d'hiver' ${ }^{5}$ ( $M V$, p. 52)

2. Pour une définition de la réduplication et une interprétation dialogique de cette figure, voir notre article "La réduplication : une interprétation dialogique " (Watine, 2012). Nous ne développons ici que les traits définitoires nécessaires à la délimitation et à l'interprétation de notre corpus.

3. Pour ce type de gémination syllabique, le terme de redoublement a d'abord prévalu, mais c'est finalement celui de réduplication qui s'impose, comme le montre le titre de l'ouvrage dirigé par Alexis Michaud et Aliyah Morgenstern en 2007. Cette plurivocité du terme réduplication provoque alors un risque de confusion entre des niveaux d'analyse hétérogènes.

4. Anna Jaubert (2011) a montré que le calembour suppose la coexistence d'un sens premier et d'un sens second plus pertinent. Dans nos exemples scatologiques, le redoublement lexical combine donc, comme les vraies réduplications qui nous occupent, le piétinement phonématique et l'adjonction d'un sens nouveau.

5. Il n'y a pas lieu d'écarter de notre corpus les réduplications après point qui doivent être considérées comme des " ajouts après point » $($ Noailly, 2002) pour lesquels le critère prosodique n'est pas déterminant. 
(3) mes habitudes, mes habitudes dominicales je veux dire. ( $M$, p. 132)

Les exemples (1) et (2) sont fondés sur une recaractérisation du substantif répété par l'adjectif qualificatif ou le complément déterminatif. Dans l'exemple (3), il s'agit d'une redétermination du nom par l'adjectif relationnel ; du reste, le marqueur de glose " je veux dire " signale l'appartenance de cette fausse réduplication aux gloses de reformulation à visée spécifiante (Niklas-Salminen \& Steuckardt, 2005) et la figure comporte donc une dimension explicitement méta-énonciative et correctrice que n'a pas la réduplication sans ajout qui nous intéresse ici.

Dans la réduplication au sens strict que nous venons de définir, aucun ajout de sens, aucune reformulation spécifiante n'est a priori possible. Quelle est donc la fonction de cette pure répétition qui semble signer le gel de toute progression microtextuelle?

\section{INTERPRÉTATION ICONIQUE ET DIALOGIQUE DE LA RÉDUPLICATION : LE PIÉTINEMENT BECKETTIEN}

\section{Interprétation iconique}

Tout comme la tautologie, la vraie réduplication combine le «tropplein discursif " avec le "vide informatif " : elle en dit plus pour ne rien dire de plus. Elle obéit donc à l'image de locuteur ressassant que les narrateurs beckettiens donnent volontiers d'eux-mêmes.

Le cadre rhétorique nous a toutefois donné le moyen de la pourvoir d'un sens : comme toute répétition, elle est susceptible d'une interprétation figurale où le paradoxe de la répétition inutile se résout dans la mise en évidence d'un effet de sens figuré. Celui-ci consisterait à faire porter un accent sur «quelques mots d'un intérêt plus marqué, ou sur lesquels la passion appuie avec plus de force " (Fontanier, 1821-1827, p. 330). Dans cette tradition, la figure a trait à l'emphase et à l'expression du haut degré. Elle a ainsi une dimension iconique : l'amplification du signifiant entraîne celle du signifié.

Certaines occurrences, dans le corpus beckettien, obéissent à cette logique d'intensification rhétorique :

(4) Le temps délicieux, délicieux, tout autre que moi s'en serait félicité. (M, p. 38)

(5) mon crâne tout ridé et crevassé et brûlant, brûlant. (p. 81)

(6) solution certes peu élégante, mais solide, solide. (p. 96)

Dans ces trois occurrences, la réduplication indique le haut degré de la qualification : on peut substituer à l'élément répété un élément

6. Parce que ces deux figures possèdent quelque similitude, nous reprenons ici les expressions que Lucile Gaudin-Bordes applique à la tautologie (Gaudin-Bordes, 2008, p. 57). 
simple assorti d'un adverbe de degré comme vraiment (le temps vraiment délicieux). Ce type d'analyse, toutefois, ne nous semble pertinent que pour les classes de mots qui, en langue, sont justement propres à varier en degré, comme l'adjectif qualificatif - ce qui ne concerne qu'un petit nombre de réduplications chez Beckett.

À côté de l'intensification, il faut ajouter la valeur, iconique elle aussi, de répétition. Lorsqu'un sème contextuel d'itération peut être repéré dans la chaîne, la figure met en acte iconiquement, dans la linéarité du texte, cette répétition même :

(7) tant de jours et de nuits donnés sans compter à cette rumeur qui se lève à la naissance et même avant, à cet insatiable Comment faire ? Comment faire ? [...]. (M, p. 11)

(8) Tout en rampant le calcul mental. [...] Genou main genou main deux. (C, p. 67)

(9) Seule elle écouta le bruit de ses pas dans l'allée, s'éloignant, se rapprochant, s'éloignant, se rapprochant. ( $M M$, p. 61)

En (7), la réduplication du discours direct nominalisé est le correspondant, sur l'axe syntagmatique, du sème itératif compris dans « insatiable ». En (8) et (9), elle est mimétique des mouvements répétitifs de la reptation ou du va-et-vient.

Dans d'autres cas enfin, c'est un sème de continuité qui est mis en acte par la linéarisation iconique :

(10) Je parle, parle, car il le faut [...]. (I, p. 33)

(11) Que d'histoires je me suis racontées, accroché au moisi, et enflant, enflant. (MM, p. 84)

(12) je me disais, Il se rapetisse, se rapetisse. ( $M$, p. 15)

Dans ces exemples, c'est l'aspect lexical progressif des verbes qui est rejoué dans la figure : on pourrait donc la remplacer par une périphrase aspectuelle. Par exemple pour (12), je me disais, Il ne cesse de se rapetisser.

Que conclure de ces premiers exemples? Dans tous ces cas de figuralité iconique, la réduplication paraît viser un effet de simplification des moyens linguistiques. On le voit dans les substitutions que nous proposons, elle évite de recourir aux adverbes intensifs, aux circonstants itératifs ou aux périphrases aspectuelles qui construiraient des rapports de dépendance syntaxique et supposeraient un apport sémantique. Elle va dans le sens d'une simple mise en série parataxique et tautologique des syntagmes. De ce point de vue, elle est le signe d'une langue répétitive et sommaire que le lecteur identifiera volontiers comme une configuration oralisante ${ }^{7}$ : elle appartient à un calque littéraire de langue

7. La plupart des études sur la réduplication constatent sa domination dans la langue parlée (voir par exemple Dostie, 2008 ; et Hammer, 1997) ; en littérature elle est souvent considérée comme l'apanage du registre enfantin ou du style parlé. 
parlée, en tant que celle-ci est souvent perçue comme une version sous-grammaticalisée de la langue. La réduction des moyens lexicaux et grammaticaux et l'iconisation du sens participent à l'élaboration d'une langue minimale propre au " paradigme vocal " (Philippe \& Piat, 2009, p. 68) qui se répand en littérature dès la fin du $\mathrm{XIX}^{\mathrm{e}}$ siècle.

\section{Interprétation dialogique}

Toutefois, l'interprétation iconique de la figure, qui ressortirait à une sommaire oralité, nous semble limitée dans le corpus beckettien. Que dire de ces nombreuses répétitions qui n'intensifient et ne miment rien ? Ainsi dans ces exemples:

(13) La chambre sentait l'ammoniaque, oh pas que l'ammoniaque, mais l'ammoniaque, l'ammoniaque. ( $M$, p. 22)

(14) j'allais dire comme dans un rêve, mais pas du tout, pas du tout. (p. 52)

Nous ferons ici l'hypothèse que ces occurrences rebelles à l'analyse iconique gagnent à être expliquées dans le cadre du dialogisme. L'exemple (13) est remarquable de ce point de vue. Il comprend une remise en question du posé initial par la rectification (" oh pas que l'ammoniaque »). Or, cette rectification négative semble constituer une concession formulée en réponse à une objection implicite qui émane du narrateur lui-même, ou qu'il imagine pouvoir être formulée par un interlocuteur. La réduplication advient alors, pour finalement confirmer le dit initial après sa remise en question supposée. On a donc le schéma : dit initial/objection (implicite)/concession ("oh pas que l'ammoniaque »)/ confirmation du dit par la réduplication. Celle-ci réagit donc à une remise en question préalable du segment par un énonciateur second; cette structure est profondément dialogique - si l'on définit le dialogisme comme "la capacité de l'énoncé à faire entendre, outre la voix de l'énonciateur, une ou plusieurs voix qui le feuillettent énonciativement » (Détrie, Siblot \& Verine, 2001, p. 83).

La fonction de réponse aux objections d'autrui nous semble confirmée par le test de l'intercalation possible d'un oui (ou d'un non en contexte négatif) : mais l'ammoniaque, oui l'ammoniaque. Ce test valide l'existence d'un dialogue implicite avec un énonciateur autre - que cet énonciateur soit réellement perçu comme autre, ou bien comme une part de soi. En effet, comme on le verra, l'objection concernant la pertinence des termes choisis peut aussi bien venir du locuteur lui-même ; d'ailleurs, pour Julien Piat, oui et non impliquent un retour du locuteur sur sa propre énonciation : "L'impression est celle d'un dialogue entre soi et soi, instauré quant à la pertinence [...] du choix des termes » (Piat, 2007, p. 122). Ces réduplications s'apparentent alors à des occurrences comme celle-ci : 
(15) à la contempler, si j'ose dire, à la contempler qui peu à peu se ratatinait $[\ldots] .(M$, p. 69$)$

C'est-à-dire qu'elles s'apparentent à des structures à décrochement méta-énonciatif avec marqueur propositionnel portant sur la pertinence $\mathrm{du}$ dire, abondantes par ailleurs dans le corpus beckettien.

Il apparaît donc que dans un grand nombre d'occurrences, la figure a peu à voir avec un mime d'oralité simplificatrice fondé sur une syntaxe et une progression minimales. Elle combine une structure dialogique qui sous-entend une contestation du dire (venue du locuteur premier, ou d'autrui) et une pesée méta-énonciative de la pertinence. Elle est donc révélatrice d'un rapport complexe du locuteur à sa langue, fondé sur une remise en question permanente de son propre dit.

\section{DEGRÉS DE LA CONFIRMATION ET ETHOS DU LOCUTEUR}

Cette remise en question n'a pas toujours la même intensité. En effet, l'étape de confirmation du dit, propre à la structure dialogique que nous venons de mettre en évidence, comporte des degrés variables. Cela fonde des figures diverses de locuteur : celui-ci peut apparaître comme plus ou moins maitre de son discours. Or ces degrés de confiance dans sa propre parole doivent être mis en relation avec les types de dialogisme mis en jeu dans la figure.

\section{Confirmation ferme}

Dans les exemples suivants, la puissance de réaffirmation du dit est élevée :

(16) à table, à table, la chambre peut attendre. ( $P A$, p. 16)

(17) Avortez, avortez, comme ça elle [l'aréole] ne foncera plus. (p. 52)

(18) Décomposer c'est vivre aussi, je le sais, je le sais, ne me fatiguez pas [...]. (M, p. 32)

Or toutes ces occurrences ressortissent au dialogisme interlocutif. En d'autres termes, elles supposent que dans la communication un interlocuteur objecte ou risque d'objecter au discours du locuteur premier ; celui-ci anticipe sur la réception de son énoncé et réaffirme le dire contesté. Cette remise en cause externe peut être clairement explicitée, par l'allocution "ne me fatiguez pas » en (18), ou par la description des actions sur le point d'être accomplies par les allocutaires : (16) peut ainsi être glosé par à table, oui à table, plutôt que de ranger la chambre. Ici la réduplication semble dire : je sais bien qu'éventuellement vous n'êtes pas d'accord avec moi, voire peu disposé à m'obéir, mais c'est tout de même mon point de vue. Elle favorise ainsi une posture de "sur-énonciation » du locuteur, c'està-dire une " expression interactionnelle d'un point de vue surplombant » 
(Rabatel, 2004, p. 9). On ne s'étonne donc pas que ces réduplications émanant d'énonciateurs sûrs de leur fait ne soient pas dominantes dans l'œuvre beckettienne. On les trouve surtout dans l'œuvre précoce (Premier amour et Molloy).

\section{Confirmation faible}

Dans beaucoup d'autres occurrences, la confirmation du dire antérieur semble bien plus incertaine :

(19) la route était en vagues, peu profondes mais suffisamment, suffisamment. (M, p. 10)

(20) Il m'est arrivé de tomber sans le vouloir, mais pas souvent, pas souvent $[\ldots]$. (I, p. 57)

(21) pour réviser les machines les apprêter pour l'aube proche. L'aube proche. (MM, p. 32)

(22) Jamais elle n'en vit faire un pas vers elle. Ou elle oublie. Elle oublie. (MV, p. 28)

(23) Enflure sans doute - enflure sans doute du joint entre phalange et phalangine $[\ldots]$. (p. 40)

Dans ces exemples, la contestation du dit ne vient pas de l'interlocuteur : elle émane du locuteur lui-même qui manifeste un doute sur la pertinence de son dit et sa fidélité au réel. Si objection il y a, elle reste confinée dans la sphère même du locuteur : la figure ressortit ici à ce que Jacqueline Authier-Revuz appelle l' " autodialogisme ", forme de " dialogue de l'énonciateur avec lui-même qui réagit à sa propre parole " (Authier-Revuz, 1995, p. 152). Le locuteur semble s'interroger sur la pertinence des termes choisis avant de les confirmer, comme faute de mieux. Dans de tels cas, la figure construit un ethos de locuteur bien plus hésitant, traversé par un doute énonciatif paralysant. Le rôle des marqueurs de ponctuation est ici significatif : le point en (21) ou (22) marque graphiquement la pause rythmique imposée par l'hésitation même, le temps nécessaire à la rétrospection évaluative sur le dit. Le tiret simple en (23) combine la pause réflexive et l'amorce de la reformulation qui suit.

\section{Réduplication de mots grammaticaux : le ratage}

Enfin, la réduplication autodialogique s'apparente parfois à une linéarisation des processus cognitifs d'élaboration mentale du discours ; la figure a alors une composante non pas rétrospective mais prospective. Ainsi :

(24) Je ne - je ne me sentais pas malheureux. ( $M$, p. 26) 
(25) J'attribuerais volontiers une partie de mes, de mes infortunes à ce désordre auditif [...]. Infortunes, bienfaits, je n'ai pas le temps de choisir mes mots [...]. (MM, p. 54-55)

(26) À force de - faillite à force de faillite la folie s'en mêle. ( $M V$, p. 37-38)

Dans ces exemples, la réduplication fournit au locuteur le temps matériel de trouver le mot recherché ; ce temps nécessaire pour « choisir [s]es mots " est d'ailleurs explicitement évoqué en (25). La répétition du mot grammatical antécédent constitue alors le signe d'une " autoreprésentation du dire en train de se faire " (Authier-Revuz, 1995, p. 18); ces réduplications marquées par la pause réflexive sont le signe d'un " ratage d'hésitation " (Barbéris \& Maurer, 1998, p. 45) normalement propre au discours oral, le temps de l'écrit préservant en principe de ces traces linéaires d'élaboration progressive du message linguistique. La présence de telles scories donne de l'écrit littéraire une image hybride et de l'énonciateur un portrait peu flatteur, le second faisant sans cesse état de ses embarras discursifs, construisant ainsi un ethos de constante incertitude.

Le fonctionnement de la figure diffère un peu des exemples précédents. Dans les termes de la praxématique, les ratages, traces des opérations de l'actualisation, manifestent le retard de la programmation linguistique (temps de l'à-dire) sur la réalisation effective de l'énoncé (temps du dire). Á cet égard, les exemples (24) à (26) doivent être distingués des exemples (13) à (23) qui révélaient plutôt l'empiètement du temps du dit (mémorisation des énoncés) sur le temps du dire. En d'autres termes, (13) à (23) relèvent d'une reconfiguration rétrospective, car ils reviennent sur un terme déjà énoncé pour peser sa pertinence ; mais (24) à (26) révèlent une configuration anticipatrice, traduisant l'attente du terme le moins impertinent. Ce dernier est dès lors dénoncé comme probablement peu adéquat : malgré un autre rapport au temps de l'énonciation, la réduplication des mots grammaticaux fonctionne elle aussi comme marqueur de mise à distance méta-énonciative, comme l'atteste la boucle réflexive qui clôt l'exemple (25).

$\mathrm{Au}$ terme de ce premier parcours, on peut noter que contrairement à l'apparence, la figure ne va jamais dans le sens d'un simple piétinement de la progression discursive. Elle comporte parfois un sens iconique visant à une simplification syntaxique et sémantique tout oralisante ; mais elle ressortit plus fréquemment au dialogisme et admet alors une valeur méta-énonciative consistant dans un retour évaluatif sur le dit. Le dit initial y est finalement confirmé, avec plus ou moins de force selon le type de dialogisme mis en jeu ; et le degré de confirmation est de plus en plus faible au cours de l'œuvre, comme si les locuteurs sûrs de leur fait en étaient progressivement exclus.

Dans les occurrences que nous allons aborder à présent, la dimension critique de la figure s'affiche plus nettement encore. 


\section{RÉDUPLICATION ET DIALOGISME DISSENSUEL : LA RÉGRESSION DISCURSIVE}

Il peut arriver que la figure soit privée de l'étape de confirmation finale. La part d'objection et de dissensus domine, le malaise des locuteurs concernant leur propre dire ne leur permet pas de dominer l'objection ou l'hésitation qui troublent la ligne énonciative. Dans de tels cas, l'intercalation de oui (ou de non en contexte négatif), qui était le signe du consensus final du locuteur à l'égard de son propre dire, devient impossible. La figure ressortit toujours au dialogisme, mais celui-ci se fait dissensuel.

\section{Citation par un autre locuteur}

Dans un premier type d'occurrences, le segment répété n'appartient plus à la même origine énonciative : il est explicitement cité par un locuteur second, dans le cadre d'une séquence dialogale :

(27) Je lui demandais s'il n'y aurait pas moyen, de temps en temps, de manger un panais. Un panais! s'écria-t-elle, comme si j'avais exprimé le désir de goûter du bébé juif. ( $P A$, p. 51)

(28) Bien papa, dit-il. Bien papa ! Ah ! (M, p. 135)

(29) Qu'est-ce que tu as regardé ? dis-je. Les liliacés, papa, répondit-il. Les liliacés papa! Il avait une façon de dire papa, mon fils, quand il voulait me blesser, très particulière. $(M$, p. 140)

De tels cas s'apparentent aux phénomènes de "reprise en écho " étudiés par Jeanne-Marie Barbéris. Pour reprendre ses termes:

[Cette] reprise en écho de nature dialogique manifeste un dissensus de la part de L2 à l'égard du propos de L1. L2 reprend le propos tenu antérieurement [...], mais il le réénonce en interprétant l'énoncé de L1 à sa manière, et en le faisant dialoguer avec son propre point de vue. (Barbéris, 2005, p. 160)

La reprise fait de l'énoncé répété un segment autonyme («Un panais!» est glosable par Vous avez bien dit un panais !), ce qui le rend apte à la caractérisation critique par le locuteur second, comme l'atteste le commentaire de Moran en (29) sur la diction même de son fils - le critère intonatif est en effet déterminant dans le repérage du dissensus. Ce segment matérialise cette fois une étape d'objection forte en contestant sans appel le dire d'autrui. Dès lors, l'énonciateur du segment répété manifeste une posture critique de sur-énonciation qui en fait le dénonciateur et le redresseur des discours autres. On ne s'étonne pas que deux de ces occurrences soient énoncées par Moran. 


\section{Auto-citation}

Il est certain que ces réduplications constituent des cas limites de la catégorie, puisque les deux segments ne sont pas énoncés par le même locuteur. Mais il est des cas proprement beckettiens, à la limite de l'acceptabilité, où ces "reprises en écho " sont bien le fait du même locuteur. On en trouve plusieurs exemples dans toute l'œuvre. Certains sont assortis d'une combinaison repérable de marqueurs graphiques :

(30) J'enlevais souvent mes chaussures quand je me couchais, je veux dire quand je me composais (composais!) à dormir [...]. (PA, p. 41)

(31) Il n’y avait qu'à (qu'à !) mettre par exemple, pour commencer, six pierres dans la poche droite de mon manteau [...]. (M, p. 96)

(32) Mais à vrai dire (à vrai dire !) je n'ai jamais été particulièrement résolu $[\ldots]$. (p. 41)

Ici, contrairement à ce qui se passait précédemment, le locuteur revient sur son propre dit, mais pour le disqualifier ; cette réaction à son propre discours est hautement dissensuelle. En effet, si l'on se réfere au schéma proposé plus haut (dit initial/objection/concession/ confirmation du dit), on peut dire qu'ici l'étape d'objection n'est pas implicitée : elle est matérialisée dans le segment rédupliqué. La réduplication n'est plus la réponse à une objection venue de soi ou d'ailleurs : elle est l'objection elle-même. En superposant linéairement le dit et sa critique par le même locuteur, la figure constitue donc ici une attaque frontale du locuteur contre son propre dit.

Dans nos exemples, elle comporte également une fonction métapoétique, car elle dit quelque chose de la faculté et de la possibilité d'écrire : l'occurrence (32) délexicalise le modalisateur d'énonciation " à vrai dire " pour le rapporter à la théorie de l'impossible vérité du récit selon Molloy ("Dire c'est inventer ", proclame-t-il à la même page). L'occurrence (31), tout en soulignant l'impertinence de la négation uniceptive au regard de l'incroyable complexité de la combinatoire des pierres et des poches exposée ici, permet un calembour scatologique qui renvoie aussi à la voix " quaqua " de Comment c'est et qui signe la dimension tout excrémentielle du discours. Quant à l'occurrence (30), elle joue sur la polysémie du verbe, feignant de souligner le caractère vieilli de la construction se composer à dormir, mais dénonçant aussi la vanité de toute composition, en particulier littéraire.

Les marqueurs graphiques ont un rôle d'appui dans le repérage de l'autodialogisme dissensuel : tandis que les parenthèses marquent le décrochage énonciatif propre au commentaire métadiscursif, le point d'exclamation est la marque du dissensus.

Après la première trilogie, on trouve encore quelques occurrences de ce type dans Mal vu mal dit, intervenant cette fois après point et sans parenthèses : 
(33) Autant dire que le sourire si c'en est un y est toujours. Ni plus ni moins. Moins! (MV, p. 63)

(34) Tel sans cesse mal vu ni plus ni moins. Moins ! (p. 72-73)

(35) Recouverte des pieds au menton d'une couverture noire elle ne livre que la tête. Ne que! (p. 48)

Ces trois exemples manifestent la même dimension opacifiante et critique que les précédents, et l'on peut également y distinguer une fonction métapoétique, la poétique beckettienne tendant progressivement à ce "moins ", ce less qui est le maître mot de Cap au pire. Dans tous les cas, ces réduplications offrent donc une critique violente de ce qu'on pourrait résumer sous le terme d'ambition littéraire.

Dans ces cas de dialogisme dissensuel, on émettra l'hypothèse qu'un type très particulier de progression discursive est à l'œuvre. En effet, de telles occurrences peuvent être interprétées comme des structures de thématisation méta-énonciative. Le segment répété dans l'exclamation devient autonyme et se lit comme le thème nouveau d'une prédication qui reste implicite dans l'énoncé exclamatif minimal. On pourrait ainsi gloser l'exemple (30) par je me composais (" composais ", quel mot!). L'autonyme, on le voit, devient le nouveau thème dans l'insertion parenthétique. De ce point de vue, ces structures réalisent des raccourcis elliptiques, situés à la limite de l'acceptabilité, de structures thématisantes autonymiques complètes : ce qui était le propos de la phrase devient dans la phrase suivante ou dans l'énoncé parenthétique un thème - ce faisant, il passe également du terme en usage à l'autonyme. Mais il s'agit d'une nouvelle prédication embryonnaire puisque le propos en est absent. En quelque sorte, l'énonciateur se contente de dénoncer l'inadéquation d'un terme, mais sans justifier sa critique, et surtout sans proposer de correction. Il se borne à la pure remise en cause du dire, comme si toute tentative d'amendement du discours était vaine...

On aurait donc ici un type de progression toute régressive, puisque l'énonciateur revient sur son dit pour le disqualifier. Le discours s'allonge à droite pour fonder une nouvelle prédication critique qui revient sur la gauche pour annuler le discours précédent, le barrer sans le corriger. Avec ce type de réduplication, l'objection méta-énonciative non combattue fonde une langue qui, localement, semble se combattre elle-même et ruiner la possibilité de la littérature : la recherche du «mal dire " passe par le dédire.

Les différents types de réduplications que nous avons tenté de mettre au jour sont révélateurs d'une constante variation dans le rapport des locuteurs à leur langue. Loin de ne mettre en œuvre qu'une stase de la progression dans un schème oralisant simplificateur, les réduplications 
complexifient la ligne énonciative en instaurant un doute sur la pertinence du dit ; mais selon le type de dialogisme qu'elles mobilisent, elles offrent une variété de postures qui s'étend de la confirmation oppositionnelle du dit à la pure invalidation non assortie de correction, en passant par la simple hésitation. Il faut souligner que cette inflation cède toutefois le pas à l'effacement de la figure dans l'œuvre tardive, où l'on observe le recul de la réduplication au profit de la répétition avec variation - ce qui nous semble le signe d'une moindre prise en charge énonciative des énoncés.

Finalement, chez Beckett, si l'on a l'air de piétiner, c'est que l'on avance à reculons : la réduplication met en scène non une répétition à l'identique mais un mouvement de retour vers le dit antérieur, que ce soit pour l'évaluer, le confirmer ou au contraire le révoquer. De ce point de vue, la progression régressive de telles réduplications nous semble répondre à une configuration générale de l'œuvre - en particulier dans les années 1950. Le retour en arrière constitue en effet un interprétant majeur, aussi bien au niveau de la structure narrative globale (celle de Molloy a souvent été analysée comme un huit de chiffre retournant sans cesse aux mêmes points) qu'au niveau de l'imaginaire (Molloy progresse vers son origine tarie, Malone tend à revivre sa naissance comme une $\operatorname{mort}^{8} \ldots$...). La réduplication est à la fois l'emblème de l'écrivain critique prenant acte des limites et de l'opacité du langage, et celui d'une configuration globale qui serait ainsi discrètement exemplifiée au niveau local. 